\title{
A REMARK ON DERIVED SPACES
}

\author{
By Tetsuhiro Shimizu
}

Let $G$ be a locally compact Abelian group, and let $\mu$ be the Haar measure on $G$. If $p \geqq 1$, the space of all $\mu$-measurable functions $f$ such that $|f|^{p}$ is summable is designated by $L_{p}(G)$.

We denote by $L_{p}^{0}(G)$ the set of all $f \in L_{p}(G)$ such that $\|f\|_{0}=\sup \left\{\|f * h\|_{p}: h \in L_{1}(G)\right.$, $\left.\|\hat{h}\|_{\infty} \leqq 1\right\}<\infty$. Clearly $L_{p}^{0}(G)$ is the linear subspace of $L_{p}(G)$. We call $L_{p}^{0}(G)$ the derived space of $L_{p}(G)$ ([5], p. 72).

The following results were showed by Helgason [3] and Figa-Talamanca [1].

Let $G$ be a locally compact Abelian group.

1) If $G$ is a compact, then $L_{p}^{0}(G)(1 \leqq p \leqq 2)$ is algebraically and topologically isomorphic to $L_{2}(G)$.

2) If $G$ is non-compact and connected, then $L_{p}^{0}(G)=\{0\} \quad(1 \leqq p<2)$.

3) If $G$ is non-compact and separable, then $L_{1}^{0}(G)=\{0\}$.

4) If $G$ is an infinite discrete group, then $L_{p}^{0}(G)=\{0\}(1 \leqq p<2)$.

We shall show the following theorem in this short note.

Theorem A. Let $G$ be a non-compact locally compact Abelian group, then $L_{p}^{0}(G)=\{0\} \quad(1 \leqq p<2)$.

Proof. From the structure theorem of locally compact Abelian groups ([6, p. 95]), we know that $G$ has an open subgroup $H$ which is the direct sum of a compact group and an Euclidean space $R^{m}$.

a) Suppose $m \geqq 1$. If we shall show that for any compact set $K$ of $G$ there exists an element $d$ of $G$ such that $\{K+k d\}, k=0, \pm 1, \pm 2, \cdots$, are pairwise disjoint, then we can prove this theorem by using the similar argument in [1].

Since $K$ is compact, there is a finite set $\left\{x_{i}: i=1,2, \cdots, n\right\}$ of $G$ such that $K \subset \cup_{i=1}^{n}\left(x_{i}+H\right)$. Put $K_{0}=\cup_{i=1}^{n}\left(\left(\left(x_{i}+H\right) \cap K\right)-x_{i}\right)$. Then $K_{0}$ is a compact subset of $H$. Since $m \geqq 1$, there is an element $d \in H$ such that $\left\{K_{0}+k d\right\}, k=0, \pm 1, \pm 2, \cdots$, are pairwise disjoint. Clearly, $\{K+k d\}, k=0, \pm 1, \pm 2, \cdots$, are also pairwise disjoint.

b) Suppose now $m=0$. Let $\dot{\mu}$ and $\mu_{0}$ be the Haar measures on $G / H$ and $H$ respectively such that

$$
\int_{G} f(x) d \mu(x)=\int_{G / H}\left(\int_{I i} f(x+y) d \mu_{0}(x)\right) d \dot{\mu}(\dot{y})
$$

Received November 12, 1970. 
and $\left\|\mu_{0}\right\|=1$. Let

$$
\Phi g(\dot{y})=\int_{H} g(x+y) d \mu_{0}(x)
$$

for each $g \in L_{p}(G)$, since $\left\|\mu_{0}\right\|=1$, we have that

$$
\begin{aligned}
& \int_{G / H}|\Phi g(\dot{y})|^{p} d \dot{\mu}(\dot{y})=\int_{G / H}\left|\int_{H} g(x+y) d \mu_{0}(x)\right|^{p} d \dot{\mu}(\dot{y}) \\
\leqq & \int_{G / H}\left(\int_{H}|g(x+y)|^{p} d \mu_{0}(x)\right) d \dot{\mu}(\dot{y})=\int_{G}|g(x)|^{p} d \mu(x) .
\end{aligned}
$$

Therefore, $\Phi$ is a norm-decreasing linear operator of $L_{p}(G)$ into $L_{p}(G / H)$. On the other hand, if $\varphi$ is the canonical homomorphism of $G$ to $G / H$, then $\Psi g(x)=g(\varphi(x))$ belongs to $L_{p}(G)$ for any $g \in L_{p}(G / H)$. Indeed,

$$
\begin{aligned}
\int_{G}|g(\varphi(x))|^{p} d \mu(x) & =\int_{G / H}\left(\int_{H}|g(\varphi(x+y))|^{p} d \mu_{0}(x)\right) d \dot{\mu}(\dot{y}) \\
& =\int_{G / H}|g(\dot{y})|^{p} d \dot{\mu}(\dot{y})<\infty .
\end{aligned}
$$

It is evident that $\Phi \Psi g=g$ for all $g \in L_{p}(G / H)$.

For $f, g \in C_{c}(G)$, let

$$
F(f, g, x ; y, \dot{z})=\int_{H} f(x+y-u-z) g(u+z) d \mu_{0}(u), \quad(x \in G, y \in H, \dot{z} \in G / H) .
$$

Suppose that $K_{g}$ is a compact support of $g$, then $F(f, g, x ; y, \dot{z})=0$ for all $z \notin\left(K_{g}+H\right)$. Since $K_{g}+H$ is compact, there exists a finite subset $\left\{z_{1}, \cdots, z_{n}\right\}$ of $G$ such that $K_{g}+H \subset \cup_{i=1}^{n}\left(z_{i}+H\right)$. Thus, we have that if $\dot{z} \notin\left\{\dot{z}_{1}, \cdots, z_{n}\right\}$, then $F(f, g$, $x ; y, \dot{z})=0$ for all $y \in H$ and $\Phi g(\dot{z})=0$. Hence, it follows that

$$
\begin{aligned}
\Phi(f * g)(\dot{x}) & =\int_{H} \int_{G} f(x+y-z) g(z) d \mu(z) d \mu_{0}(y) \\
& =\int_{H} \int_{G / H} \int_{H} f(x+y-z-u) g(z+u) d \mu_{0}(u) d \dot{\mu}(\dot{z}) d \mu_{0}(y) \\
& =\int_{H} \sum_{i=1}^{n} F\left(f, g, x ; y, \dot{z}_{\imath}\right) d \mu_{0}(y) \\
& =\sum_{i=1}^{n} \int_{H} F\left(f, g, x ; y, \dot{z}_{\imath}\right) d \mu_{0}(y) \\
& =\sum_{i=1}^{n} \int_{H} \int_{H} f\left(x+y-z_{i}-u\right) g\left(z_{i}+u\right) d \mu_{0}(u) d_{0} \mu(y) \\
& =\sum_{i=1}^{n} \int_{H} \int_{H} f\left(x+y-z_{i}-u\right) g\left(z_{i}+u\right) d \mu_{0}(y) d \mu_{0}(u)
\end{aligned}
$$




$$
\begin{aligned}
& =\sum_{i=1}^{n} \int_{H} f\left(x+y-z_{i}-u\right) d \mu_{0}(y) \int_{H} g\left(z_{i}+u\right) d \mu_{0}(u) \\
& =\sum_{i=1}^{n} \Phi f\left(\dot{x}-\dot{z}_{\imath}\right) \Phi g\left(\dot{z}_{\imath}\right) \\
& =\int_{G / H} \Phi f(\dot{x}-\dot{z}) \Phi g(\dot{z}) d \dot{\mu}(\dot{z}) \\
& =\Phi f * \Phi g(\dot{x}) .
\end{aligned}
$$

Since $C_{c}(G)$ is dense in $L_{p}(G)(1 \leqq p<2)$, we have that if $f \in L_{p}(G)$ and $g \in L_{1}(G)$, then $\Phi(f * g)=\Phi f * \Phi g$.

Let $\Gamma$ be the dual group of $G$, and let $\Lambda$ be the anihilator of $H$. For any $h \in L_{1}(G / H)$, it is evident that $\widehat{\Psi h}(\gamma)=\hat{h}(\gamma)$ for any $\gamma \in \Lambda$ and $\widehat{\Psi h}(\gamma)=0$ for any $\gamma \in \Gamma \backslash \Lambda$. Therefore, if $f \in L_{p}^{0}(G)$ and $h \in L_{1}(G / H)$, then

$$
\|\Phi f * h\|_{p}=\|\Phi(f * \Psi h)\|_{p} \leqq\|f * \Psi h\|_{p} \leqq\|f\|_{0}\|\widehat{\Psi h}\|_{\infty}=\|f\|_{0}\|\hat{h}\|_{\infty} .
$$

Consequently, $\Phi f \in L_{p}^{0}(G / H)$ for any $f \in L_{p}^{0}(G)$.

Let $f_{\gamma}(x)=(-x, \gamma) f(x)$ for any $f \in L_{p}(G)$ and $\gamma \in \Gamma$. Clearly, if $f \in L_{p}^{0}(G)$, then $f_{\gamma} \in L_{p}^{\circ}(G)$. Suppose that there exists a non-zero element $f \in L_{p}^{0}(G)$. If $\gamma$ is an element of $\Gamma$ such that $\hat{f}(\gamma) \neq 0$, then

$$
\begin{aligned}
\widehat{\Phi f}_{\gamma}(0) & =\int_{G / H} \Phi f_{\gamma}(\dot{x}) d \dot{\mu}(\dot{x}) \\
& =\int_{G / H} \int_{H}(-(x+y), \gamma) f(x+y) d \mu_{0}(y) d \dot{\mu}(\dot{x})=\hat{f}(\gamma) \neq 0 .
\end{aligned}
$$

Thus, we have that $L_{p}^{0}(G / H) \neq\{0\}$. But, since $G / H$ is infinite discrete, this is impossible. Therefore, $L_{p}^{0}(G)=\{0\}$. This completes the proof.

The following theorem was proved by Gaudry in the case of a locally compact Abelian group with an infinite discrete subgroup.

Theorem B. Let $G$ be a non-compact locally compact Abelian group. If $g$ is a function on $\Gamma$ such that $\varphi g \in \cup_{1 \leqq p<2} L_{p}(G)^{\wedge}$, where $L_{p}(G)^{\wedge}=\left\{\hat{f}: f \in L_{p}(G), \hat{f}\right.$ is the Fourier transform of $f\}$, for each $\varphi \in C_{0}(\Gamma)$, then $g$ is zero locally almost evyeywhere.

Proof. From the hypothesis, we can assume $g$ has a compact support $K$. Then, there is a number $p_{0}, 1<p_{0}<2$, such that $\varphi g \in L_{p_{0}}(G)^{\wedge}$ for all $\varphi \in C_{0}(I)$ ([2], p. 486). Let $\varphi_{0} \in C_{0}(\Gamma)$ such that $\varphi_{0} \equiv 1$ on $K$, then $\varphi_{0} g=g \in L_{p_{0}}(G)^{\wedge}$. Let $f \in L_{p_{0}}(G)$ such that $\hat{f}=g$ locally almost everywhere. Then $f \in L_{p_{0}}^{\circ}(G)([1])$. Therefore, theorem A shows $f$ is zero. Thus $g$ is zero locally almost everywhere. This completes the proof.

For $s \in G, \tau_{s}$ will denote the translation operator defined by $\left(\tau_{s} f\right)(t)=f\left(t s^{-1}\right)$. A continuous linear operator $T$ from $L_{p}(G)$ to $L_{p}(G)$ is called a multiplier for $L_{p}(G)$ whenever $T \tau_{s}=\tau_{s} T$ for each $s \in G$. The collection of all mutipliers for $L_{p}(G)$ will 
be denoted by $M\left(L_{p}(G)\right)$.

Combining the theorem A with the proof of theorem 5 in [1] we can prove the next theorem.

TheOREM C. Let $G$ be a non-compact locally compact Abelian group and suppose $1<p<\infty, p \neq 2$. If $\varphi \in L_{\infty}(\Gamma)$ corresponds a multiplier $T$ in $M\left(L_{p}(G)\right.$ ) (i.e. $(\widehat{T f})=\varphi \hat{f}$ and has the property that whenever $\phi$ is a function for which $|\psi(\gamma)|$ $\leqq|\varphi(\gamma)|$ for each $\gamma \in \Gamma$ then $\phi$ corresponds to a multiplier in $M\left(L_{p}(G)\right)$, then $\varphi=0$.

\section{REFERENCES}

[1] Figa-Talamanca, A., On the subspace of $L_{p}$ invariant under multiplication of transforms by bounded continuous functions. Rend. del Sem. Mat. Univ. Padova 35 (1965), 176-189.

[2] Gaudry, G. I., Mutipliers of type $(p, q)$. Pacific J. Math. 18 (1966), 477-488.

[3] Helgason, S., Multipliers of Banach algebras. Ann. of Math. 64 (1959), 240-254.

[4] Hörmander, L., Estimates for translation invarıant operator in $L_{p}$ space. Acta Math. 104 (1960), 93-140.

[5] LARSEN, L., The multiplier problem. Springer-Verlag, New York (1969).

[6] Rudin, W., Fourier analysis on groups. Interscience, New York (1962).

Department of Mathematics,

Tokyo Institute of Tecinology. 